\section{RECIPE FOR SUCCESS}

The editor's choice this month would make a great choice for your journal club. When you have read it and the accompanying editorial by Lamia Soghier and Billie Lou Short you will want plenty of your colleagues to read the two articles too and start talking about them. Joseph Kaempf and colleagues try to get to the bottom of what might explain some of the variations in outcomes observed between neonatal units. This takes us a step further from potentially better practices to potentially better characteristics - the features that might make some units more successful than others at implementing otherwise similar quality initiatives. There is a lot of work in this. They measured the outcomes of a large group of neonatal units over a 14 year period using a composite morbidity measure. All the units were successful at reducing their composite morbidities over the study period but some consistently outperformed the others throughout. They also conducted an environmental survey at each NICU that explored 103 unit characteristics This included 39 items related to quality improvement methodology and medical therapies, 22 related to staffing, eight related to the physical environment, 30 cultural, social and cognitive characteristics and four sham questions. They were able to identify those attributes among the 103 that were positively and negatively associated with the highest performing units. See pages F4 and F13.

\section{NETWORK META-ANALYSIS EXPLAINED}

If your journal club has any energy left after the above feast then this leading article by Sofia Dias will also be popular. Network Meta-analyses feature increasingly in evidence evaluations and developing greater understanding of this complex tool is time well spent. See pages F8.

\section{EARLY LOW DOSE HYDROCORTISONE}

Olivier Baud and colleagues report a pre-specified secondary analysis of the neurodevelopmental outcomes of infants in the PREMILOC Trial (doi: 10.1016/S01406736(16)00202-6) according to their gestation at birth. The trial showed that early low dose hydrocortisone treatment of infants born before 28 weeks gestation improved survival without bronchopulmonary dysplasia. There was no adverse effect of the treatment on neurodevelopmental outcomes overall. In their paper in this issue they report sub-group analyses for neurodevelopmental outcome in the less mature (24-25 weeks gestation) and more mature (26 and 27 weeks gestation) infants. The analysis is exploratory and was not powered to detect a significant difference in the treatment effect between gestation sub-groups. Early low dose hydrocortisone was not associated with an effect on neurodevelopmental impairment in the more mature infants. In the more immature infants moderate to severe neurodevelopmental impairment was observed less frequently in the infants who received hydrocortisone treatment. This is somewhat reassuring because an individual patient data meta-analysis of trials of early hydrocortisone published on line this month, (doi: 10.1016/S0140-6736(16)00202-6) which includes these data, shows a reduction in the risk of mortality with this treatment but an increase in the risk of late onset sepsis. It will be interesting to see how the neonatal community responds to the emerging evidence about this treatment. Time for some rapid responses? See pages F30.

\section{NEURODEVELOPMENTAL FOLLOW-UP OF SURGICAL NEONATES}

Does your NICU follow-up programme include the infants who had surgery for congenital anomalies in the population who you arrange 2 year assessments for? Annelieke Hijkoop and colleagues report 2 year outcomes for infants with omphalocoele and Karen Walker and Andrew Holland place this information in context with other reports in their accompanying editorial, recommending routine neurodevelopmental follow-up for all children with abdominal wall defects. See pages F2 and F18.

\section{ASSESSING THE RESPONSE TO RESUSCITATION}

There are many pitfalls in the assessment of the response to neonatal resuscitation, particularly in infants with very low cardiac output. No exhaled $\mathrm{CO}_{2}$ signal doesn't necessarily mean that the tube is in the wrong place. An ECG heart rate doesn't guarantee a useful cardiac output. In this issue Maria Liza Espinoza and colleagues report the time taken for the heart rate to improve above 100 beats per minute after effective ventilation in newborn piglets who had been asphyxiated to a low cardiac output state. The animals were intubated via tracheostomy with a leak free system and had direct measurements of tidal volume at the airway so there is no question that effective ventilation was provided from the outset. With $30 \mathrm{~s}$ of effective ventilation only $6 / 30$ animals had a rise in heart rate over 100. A third of animals showed any increase in heart rate in the first $15 \mathrm{~s}$ and just under half had shown no improvement in $30 \mathrm{~s}$. Contrary to received wisdom there may not be an immediate improvement in heart rate with the onset of effective ventilation after asphyxia. See pages F98.

\section{VOLUME TARGETED VENTILATION}

A review article on this topic by Martin Keszler is a welcome resource for staff getting to grips with this important advance in neonatal ventilator technology. A study of work of breathing at different targeted tidal volumes in infants with bronchopulmonary dysplasia by Anne Greenough and colleagues illustrates Keszler's point that one size does not fit all with this ventilation mode. See pages F46 and F108.

\section{POST-HAEMORRHAGIC VENTRICULAR DILATATION TRIAL}

The ELVIS trial, reported here by Linda de Vries and colleagues compared different thresholds for intervention in infants developing post-haemorrhagic ventricular dilatation. Intervention began at a low threshold (ventricular index (VI) greater than the 97th percentile (p97) and anterior horn width (AHW) $>6 \mathrm{~mm}$ ), or at a higher threshold $(\mathrm{VI}>\mathrm{p} 97+4 \mathrm{~mm}$ and AHW $>10 \mathrm{~mm}$ ). There was not a significant difference in the risk of the primary composite outcome of ventriculoperitoneal shunting or death. Infants treated at the lower threshold received more lumbar punctures and reservoir insertions. The authors remind us that it is important to remember the lessons from the DRIFT trial and to await long term follow-up because it may yet be demonstrated that earlier intervention influences later outcome. See pages F70. 